\title{
Study of Distribution Model under Disaster Relief Logistics System
}

\author{
Lingling-Chen ${ }^{1,2}$, Jiaqi-Yang ${ }^{1}$ and Jianjun-Liu ${ }^{2}$ \\ ${ }^{1}$ Wuhan university of technology, Wu han, China, 430000 \\ ${ }^{2}$ Yanching Institute of Technology, Langfang, China, 063000 \\ (Corresponding author: Jianjun-Liu, 108446270@qq.com)
}

\begin{abstract}
This paper mainly using the supplies distribution model in emergency situation, conducted a in-depth research on the problem of supplies distribution in the disaster relief, including single supplies and multiple supplies respectively with and without transport capacity constraints, and then implement its calculation through computer program; next, emergency supplies distribution model of multiple target and dynamic conditions is discussed in further, that is to say, considering some conditions such as the road condition change in the existing logistics system when disasters occur, in this case, the disaster relief supplies distribution model of object-oriented is introduced to guarantee the supplies. At present, there is still no systematic disaster relief logistics system in our country, so the study results of this paper have a certain reference value to the build and implementation of disaster relief logistic system in our country.
\end{abstract}

Keywords: disaster relief logistics system, supplies distribution model, natural disasters, emergency logistics

\section{Introduction}

Our country is a country with frequent natural disasters. Under the condition of existing manpower, material resources, financial resources and technical, losses from natural disasters are inevitable. In this case, what we can do is to make sufficient preparations and actively respond to disasters so as to reduce or eliminate the impact that hazards have on the city as far as possible.

Under the current conditions, the national disaster relief is still short of specific administration section, and in most cases, it depends on administrative means. However, the comprehensive national strength directly lead to the feasible supply of relief materials relatively limited, the lack of relevant measures and means seriously influence and restrict the distribution efficiency of disaster relief materials. Since the supply of materials is certainly one of the extremely important content in the disaster relief, it is of great importance and necessity to establish an effective disaster relief logistics system which can adopt the relatively economic scheduling mode and then accurately allocate and transport limited relief materials to disaster area through the optimal distribution process and distribution strategy in the shortest possible time. This job should also be the research focus of logistics planning department. By this token, studying disaster relief logistics system and its implementation not only is of great social significance, but also has good research value. For this purpose, this article will firstly structure the institutional framework and functional system of disaster relief logistics supplies allocate and transport system in our country; secondly, combining the two main factors in supplies allocate and transport, time and route choice problem, building model and test simulation experiment, 
in order to provide a reference for disaster prevention and reduction in the medium-sized city in China.

\section{Introduction of Disaster Relief Logistics}

\subsection{Overview of Disaster Relief Logistics}

Logistics generally refers to the flow process of supplies, starting from supply place to receiving place. While, emergency logistics is mainly to provide a kind of emergency security in the face of emergencies, such as guarantee the demand of manpower, material resources and financial resources during the process of sudden natural disasters, sudden infectious diseases as well as the public safety incident. Disaster relief logistics belongs to a kind of emergency logistics.

\subsection{Characteristics of Disaster Relief Logistics}

During the process of disaster relief, all circulation activities about supplies and materials belong to disaster relief logistics. Its features mainly include:

1) Ambient environment

Due to the sudden of incident, the environment of disaster relief changes along with the change of factors such as time and place. The difficulties in predicting and controlling lead to a sea of troubles in the process of disaster relief even faced the threat of security. As a result, the distribution of logistics must be flexible and changeable enough to cope with the complex environment of disaster relief. When disaster occurs, it is necessary to call and distribute available resources flexibly to ensure the effective utilization of supplies.

2) Organizer of disaster relief

When natural disaster or non-artificial disaster occurs, the main organizer of disaster relief should be the government, and aid from other countries as well as organizations. If the disaster is relatively serious, such as the local earthquake, the organizers also include voluntary masses and local leaders group.

3) The supply of disaster relief supplies

Once the disaster occurs, disaster relief supplies should be distributed to the disaster sites urgently. For fear of causing confusion, an effective transportation planning should be quickly formed combining with the local traffic conditions. The difference between disaster relief logistics and general logistics is shown in table 1.

Table 1. Comparison of Disaster Relief Logistics and General Logistics System

\begin{tabular}{|c|c|c|}
\hline Project & General Logistics & Disaster relief logistics \\
\hline System target & Profit maximization & Fairness and efficiency \\
\hline Planning process & Long, medium and short term & $\begin{array}{l}\text { The urgency is strong, the } \\
\text { decision-making time is short }\end{array}$ \\
\hline Facility characteristics & Permanent character & Temporary \\
\hline Location allocation model & Round and round distribution & Round trip distribution \\
\hline
\end{tabular}

4) The particularity of the supply chain

Due to the sudden of disaster, supplies providers cannot make sufficient preparation leading to the way of providing supplies very passive. Besides, the information peer-topeer between the supplier and the demand site makes the disaster relief supplies distributing center playing its core role by connecting the supplier and the demand site, at 
the same time, averting encumbrance in other links, as a result, promoting the timeliness of disaster relief logistics.

The supply chain comparison of disaster relief logistics and general logistics is shown in figure 1 .

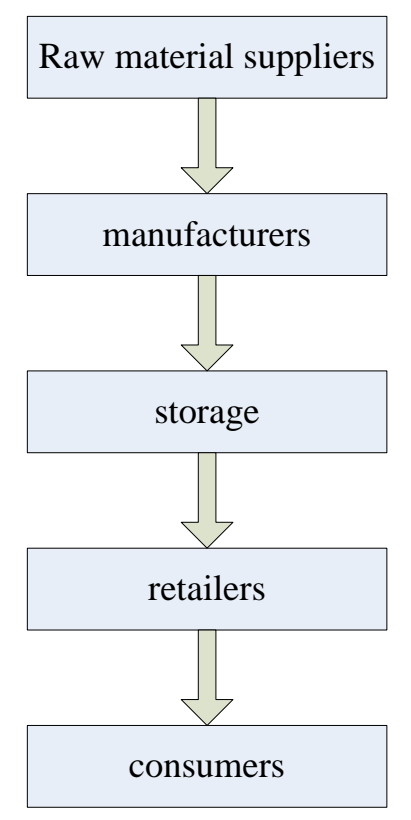

General enterprise logistics

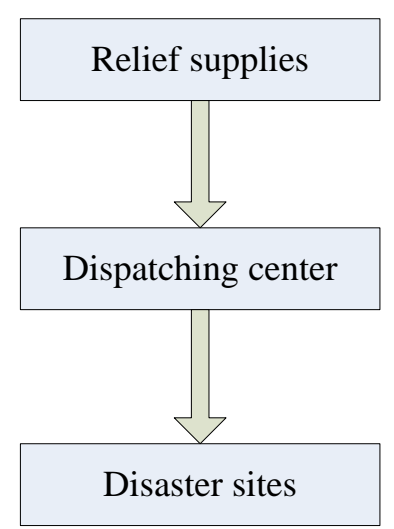

Disaster relief logistics

\section{Figure 1. The Comparison Diagram of Enterprise Logistics and Disaster Relief Logistics Supply Chain}

\subsection{Framework of supplies distribution system of disaster relief logistics}

Logistics generally refers to the flow process of supplies, starting from supply place to receiving place. While, emergency logistics is mainly to provide a kind of emergency. The relationship between supplier and demand site is relatively complex for the following reasons: first, disaster relief supplies distributing center need to quickly understand the condition of the disaster areas, estimate the quantity demanded for supplies and then conduct clustering grouping according to the quantity; Second, disaster relief supplies distributing center confirms the quantity of disaster relief supplies they can obtain from the perspective of supply; finally, adjust the supply and demand on the basis of the quantity of disaster relief supplies, try to make the supply and demand of all supplies achieve a balanced status in order to realize the scientific and reasonable distribution. Unbalanced supply and demand supplies should be distributed effectively according to the priorities of event.

After the entire process above, disaster relief supplies distributing center should obtain information repeatedly, confirm the supply and demand again in order to make a new plan at any time to meet the demand of disaster relief. The whole system framework is shown in figure 2. 


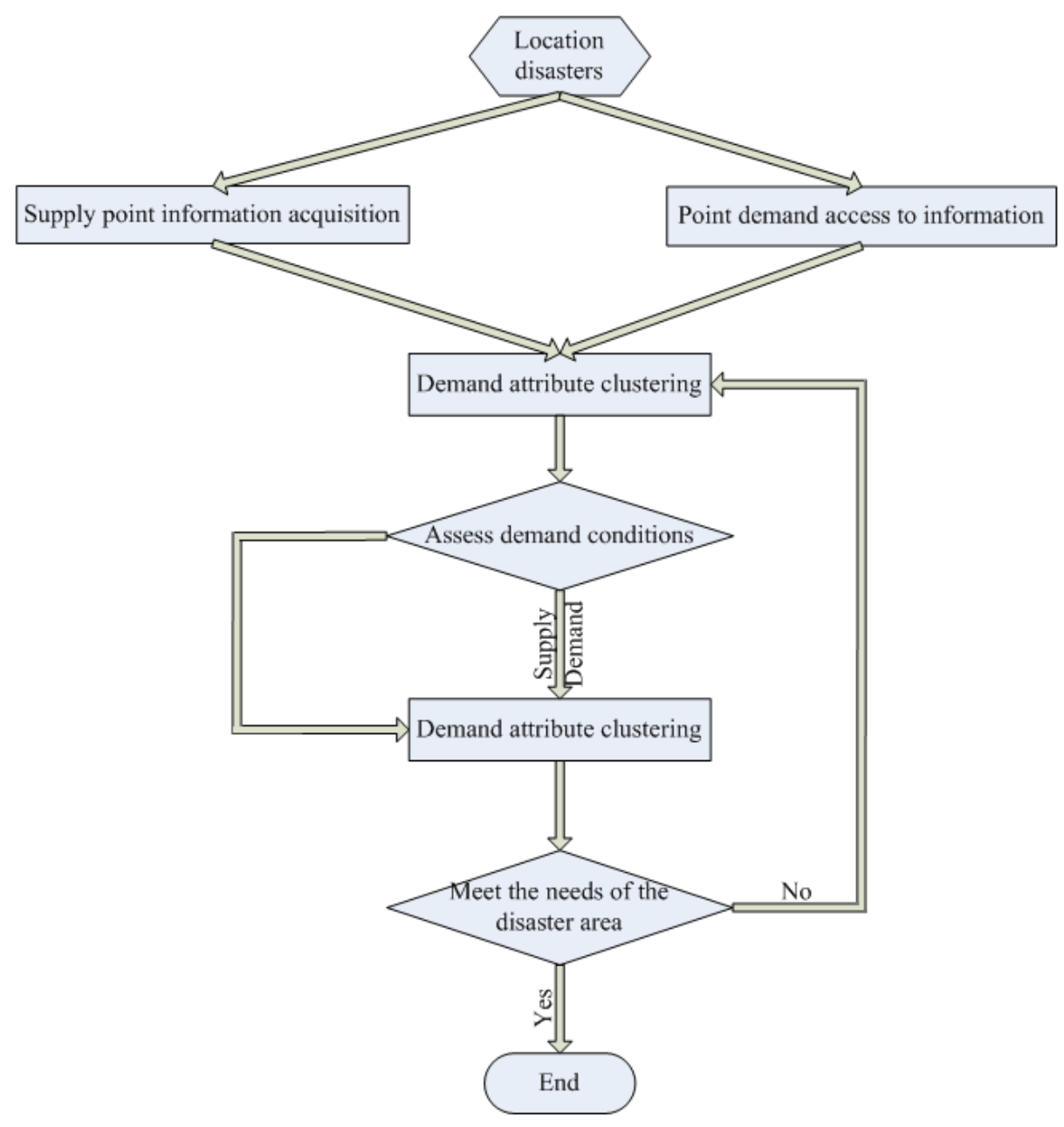

Figure 2. The Framework of Disaster Relief Logistics Distribution System

\section{Build Supplies Distribution Model}

\subsection{Theoretical Basis}

Generally, disaster relief supplies distributing should employ the nearby principle, that is to provide the supplies from the nearest for supply. But actually, supplies are limited. Once in the face of large disaster, limited supplies cannot provide enough supplies, in this case, multiple supplies suppliers are needed to transport disaster relief supplies together, let all the suppliers form an optimized combination, and at the same time satisfy the prescribed time limit. Finally, the optimal solution is received under the constraints of the above conditions.

\subsubsection{The Structure of the Mathematical Model}

Suppose $P_{1}, P_{2}, \ldots, P_{n}$ are $\mathrm{n}$ suppliers of emergency supplies, $Q$ is emergency supplies demand side. $X_{i}(>0)$ is the emergency supplies storage capacity of $P_{i}(i=1,2, \mathrm{~L}, n) . X$ is the emergency supplies quantity demanded of $Q$, and meet the condition: $\sum_{i=1}^{n} X_{i} \geq X$. Suppose the time of $P$ to $Q$ is 
$t_{i}(>0), i=1,2, \cdots, n ; T(>0)$ is the restricted period of emergency distributing time ruled in state of emergency.

As it is known to all that the allocate and transport scheme over the restricted period is void, so a supplies emergency allocate and transport scheme is urgently needed to make the time shortest under the condition of meeting the restricted period.

Suppose $\phi$ is an allocate and transport scheme of arbitrary selecting emergency supplies suppliers, $P_{1}, P_{2}, \ldots, P_{n}$ are the chosen emergency supplies suppliers, $t_{1}, t_{2}, \cdots, t_{m}$ are the corresponding emergency distributing time, $X_{1}, X_{2}, \cdots, X_{m}$ are the corresponding emergency supplies storage capacity, among them, $1,2, \cdots, m$ is one sub arrangement of $1,2, \cdots, n$.

Related definitions are as follows:

Definition 1 Emergency supplies distribution time refers to the time from giving the emergency supplies distribution orders to completing the supplies distribution job. For one distribution scheme, the corresponding distribution time is $T(\phi)$.

Definition 2 A feasible distribution scheme means: the emergency supplies storage capacity of each supplies supplier in this distribution scheme $X_{1}, X_{2}, \cdots, X_{m}$ meet

$$
\sum_{i=1}^{m} X_{i} \geq X
$$

Definition 3 Define $\chi$ is the set of all distribution scheme, $\chi^{1}$ is the set of all feasible of feasible distribution scheme, it is clearly that $\chi^{1} \subset \chi$.

Due to each of the supplies suppliers emergency distribution id conducted at the same time, so for this kind combination emergency scheme which involved in multiple suppliers, the emergency distribution time has the following properties:

Property 1

$$
T(\phi)=\max _{i=1,2, \cdots, m} t_{i}
$$

Namely the completing time of emergency supplies distribution scheme is the time of the last car experienced arriving at the emergency supplies demand site. Based on objectives and constraints of emergency distribution, we can construct a mathematical model with emergency distribution shortest as the only target.

$$
\begin{gathered}
\min T(\phi)=\min \max _{i=1,2, \cdots, m} t_{i} \\
\text { s.t }\left\{\begin{array}{l}
\sum_{i=1}^{m} X_{i} \geq X \\
t_{i} \leq T
\end{array}\right.
\end{gathered}
$$

By definitions and properties, above model can be simplified as:

$$
\min _{\phi \in \chi^{1}} T(\phi)
$$


Our goal is to find out the solution $\phi^{*}$ satisfying the constraint conditions, make $\min T\left(\phi^{*}\right)=\min T(\phi)$. Next are the solving process of 3.3 and 3.4 mathematical model.

\subsubsection{Solution of the Single Target Mathematical Model}

To not lose generality, assume $t_{1} \leq t_{2} \leq \cdots t_{n} \leq T$, the following discussion will based on this. For example, if the time of one emergency supplies supplier to emergency supplies demand site more than $\mathrm{T}$, then this supplier cannot participate in emergency distribution.

Define 4 for distribution scheme $\phi=\left\{\left(P_{1}, X_{1}\right),\left(P_{2}, X_{2}\right), \cdots,\left(P_{m}, X_{m}\right)\right\}$, among which $1,2, \cdots, m$ is one of the sub arrangement of $1,2, \cdots, n$. Suppose $t_{1} \leq t_{2} \leq \cdots t_{m} \leq T$ to sequence $X_{1}, X_{2}, \cdots, X_{m}$ has $k$, satisfying $1 \leq k \leq m \leq n$, make $\sum_{i=1}^{k-1} X_{i}<X \leq \sum_{i=1}^{k} X_{i}$, then $k$ is the critical subscript of this sequence relative to $X$.

Lemma the sufficient and necessary conditions of feasible scheme is that there exist the critical subscript relative to $X$ in sequence $X_{1}, X_{2}, \cdots, X_{m}$.

\section{Prove}

Necessity: if the scheme $\phi$ is feasible, that is $\sum_{i=1}^{m} X_{i} \geq X$, then exist $k_{s}$, satisfying $1 \leq k_{s} \leq m \leq n \quad$, making $\sum_{i=1}^{m} X_{i} \geq X \quad$, take $k=\min \left\{k_{s}\right\}$, then $\sum_{i=1}^{k-1} X_{i}<X \leq \sum_{i=1}^{k} X_{i}$. By the definition of critical subscript, in the sequence $X_{1}, X_{2}, \cdots, X_{m}$, there exist critical subscripts $k$ relative to $X$.

Sufficiency: If there exist critical subscript relative to $X$ in sequence $X_{1}, X_{2}, \cdots, X_{m} \quad$ namely, exist $k$ satisfying $1 \leq k_{s} \leq m \leq n$ making $\sum_{i=1}^{k-1} X_{i}<X \leq \sum_{i=1}^{k} X_{i}$. As a result, there must be $\sum_{i=1}^{m} X_{i} \geq X$, in other words, scheme $\phi$ is feasible.

If $k$ is the critical subscript relative to $X$ in sequence $X_{1}, X_{2}, \cdots, X_{m}$, then the distribution scheme which take $P_{1}, P_{2}, \ldots, P_{k}$ as supplies supplier makes the best target and make $T\left(\phi^{*}\right)=\max _{i=1,2, \cdots, k} t_{i}=t_{k}$. The characteristics of this scheme are: choosing the emergency supplies supplier $P_{1}$ which is the nearest to emergency supplies demand site to participate in emergency distribution. If all of its emergency supplies storage capacity $X_{1}$ is less than the total demand quantity of emergency supplies $X$, in this case, make the emergency supplies supplier $P_{2}$ which is the second nearest to the emergency supplies demand site o participate in emergency distribution. If the total emergency supplies storage capacity of $P_{1}$ and $P_{2}$, $X_{1}+X_{2}$, is still less than the total demand quantity of emergency supplies $X$, in this case, make the emergency supplies supplier $P 3$ which is the third nearest to the emergency supplies demand site o participate in emergency distribution and so 
on, until satisfying the demand of emergency supplies. For this, there are the following theorem and corollary:

Theorem 1 The distribution schemes $\phi^{*}$ which takes $P_{1}, P_{2}, \ldots, P_{k}$ as emergency supplies supplier will make the optimal target. $k$ is the critical subscript relative to $X$ in the sequence $X_{1}, X_{2}, \cdots, X_{n}$, at the same time, there has $T\left(\phi^{*}\right)=\max _{i=1,2, \cdots, k} t_{i}=t_{k}$.

The prove process can be concluded from the above analysis.

Corollary Suppose $\varphi$ is the optimal solution of the single objective in 3.3 or 3.5. For another solution $\phi$, if satisfying $T(\varphi)<T\left(\phi^{*}\right)=t_{k}$, then we can get: $\phi$ must not be feasible.

\subsubsection{Computer Implementation of the Solving Process}

According to the solution thinking above, the model can be solved by the corresponding computer program.

\subsection{Build of Model}

\subsubsection{Hypothesis of condition}

1) Suppose $P_{1}, P_{2}, \ldots, P_{n}$ are the $\mathrm{n}$ emergency supplies suppliers under emergency situations, $Q$ is the emergency supplies demand site;

2) In state of emergency, demand site $Q$ needs $m(m>1)$ kinds of emergency supplies in total, suppose $X=\left(X_{1}, X_{2}, \cdots, X_{m}\right)$ stands for emergency supplies demand quantity, where $X_{j}$ is the demand for $j$ supplies, and $j=1,2, \cdots, m$;

3) Suppose the max transport quantity of $j$ supplies in restrict time is $X_{i j}, i=1,2, \cdots, n ; j=1,2, \cdots, m$; and satisfy $\sum_{i=1}^{n} X_{i j} \geq X_{j}$. Besides, for any $A_{i}$, there are $X_{i j} \geq 0$ and not all are 0 ;

4) Assume that the transport capacity of each $j$ supply is known and unrelated;

5) Set the time of distributing maximum quantity $X_{i j}$ from $P_{i}$ to $Q$ is $t(>0), i=1,2, \cdots, n ; T(>0)$ is the restricted period of emergency distribution time ruled in state of emergency.

To not lose generality, we assume $t_{1} \leq t_{2} \leq \cdots \leq t_{n} \leq T$, if the time from emergency supplies supplier to the emergency supplies demand site is more than the restricted period $T$, then this emergency supplies supplier cannot participate in emergency transportation. Now, it is required to give a solution, which can make sure the emergency supplies suppliers participating in emergency transportation and the supplies quantity of each supplier, to make the emergency distribution time shortest under the condition of time limit.

\subsubsection{Single Objective Model Including Transport Capacity Constraints}

When $\mathrm{m}=1$, namely single supplies emergency distribution, this kind of problem has been solved in the previous section;

When $m>1$, we will generalize the solution on the basis of the last section to the situation of to multiple supplies emergency distribution.

Because is multiple supplies with a variety of supplies involved in emergency distribution, so any one distribution scheme can be expressed by the matrix as: 


$$
\phi=\left(\begin{array}{cccc}
X_{11}^{\prime} & X_{12}^{\prime} & \cdots & X_{1 m}^{\prime} \\
X_{21}^{\prime} & X_{22}^{\prime} & \cdots & X_{2 m}^{\prime} \\
\cdots & \cdots & \cdots & \cdots \\
X_{n 1}^{\prime} & X_{n 2}^{\prime} & \cdots & X_{n m}^{\prime}
\end{array}\right)
$$

Among them, $0 \leq X^{\prime}{ }_{i j} \leq X_{i j}, \sum_{i=1}^{n} X^{\prime}{ }_{i j}=X_{j}, j=1,2, \cdots, m ; i=1,2, \cdots, n$.

$X_{i j}$ stands for the largest distribution quantity of $j$ supplies in restrict period $P_{i}$, $X_{i j}^{\prime}$ stands for the actual distribution quantity of $j$ supplies when $P_{i}$ supplier participate in with scheme $\phi$. It can be seen from scheme $\phi$ that line $i$ stands for the supplies emergency distribution quantity of $P_{i}$ supplier, column $j$ stands for the distribution quantity of each supplier participating of $j$ supplies. Here can be described as:

$$
\phi=\left(\begin{array}{c}
\phi^{1} \\
\phi^{2} \\
\vdots \\
\phi^{n}
\end{array}\right)=\left(\begin{array}{llll}
\phi_{1}, & \phi_{2}, & \cdots & \phi_{m}
\end{array}\right)
$$

Among them, $\phi^{i}, i=1,2, \cdots, n$ stands for the distribution supply scheme of each supply in supplier $P_{i} ; \phi_{j}, i=1,2, \cdots, m$ stands for the distribution scheme of $j$ supplies in demand site $P$.

Property $1 \phi^{i}, i=1,2, \cdots, n$ satisfies $\phi^{i} \geq 0$. If $\phi^{i}=0$, it means that this emergency supplies supplier $P_{i}$ don't participate in emergency distribution.

Property $2 \phi_{j}, i=1,2, \cdots, m$ satisfies $\phi_{j} \geq 0$, and there exist one $i$, make $X_{i j}^{\prime}>0$. $\phi_{j}$ is the distribution scheme of $j$ emergency supplies, the it can be also expressed as:

$\phi_{j}=\left\{\left(P_{1}, X^{\prime}{ }_{1 j}\right),\left(P_{2}, X^{\prime}{ }_{2 j}\right), \cdots,\left(P_{n}, X_{n j}^{\prime}\right)\right\}$

Allowing for this paper generalize the solution on the basis of the last section to the situation of to multiple supplies emergency distribution, the following discussion only consider notation:

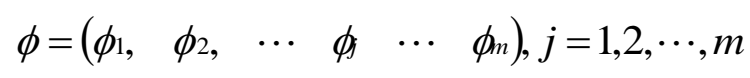

The next step is to build the multiple supplies emergency distribution model which takes "emergency distribution time shortest" as target. When only consider $j$ supplies, $\phi_{j}=\left\{\left(P_{1}, X^{\prime}{ }_{1 j}\right),\left(P_{2}, X^{\prime}{ }_{2 j}\right), \cdots,\left(P_{n}, X^{\prime}{ }_{n j}\right)\right\}$. Then exclude the point $X_{i j}^{\prime}=0$ in supplier $P_{i}$, and rearrange them, get:

$$
\phi_{j}=\left\{\left(P_{i_{1}}, X_{i_{2} j}^{\prime}\right),\left(P_{i_{2}}, X_{i_{2}}^{\prime}\right), \cdots,\left(P_{i_{q}}, X_{i_{q}}^{\prime}\right)\right\}
$$

Among them, $i_{1}, i_{2}, \cdots, i_{q}$ is an arrangement of sub sequence $1,2, \cdots, n$. By the definition and model in 3.2, get: 


$$
\begin{array}{r}
\min T\left(\phi_{j}\right)=\min \max _{i=1,2, \cdots, q} t_{i j} \\
\text { s.t }\left\{\begin{array}{l}
\sum_{k=1}^{q} X_{i_{k} j} \geq X_{j} \\
t_{i_{k}} \leq T
\end{array}\right.
\end{array}
$$

According to model 3.7, the best distribution scheme satisfying time shortest for each supply can be solved:

$\phi_{1}, \quad \phi_{2}, \cdots, \quad \phi_{j}, \cdots, \quad \phi_{m}$,

The distribution scheme of multiple supplies is:

$$
\phi=\left\{\phi_{1}, \quad \phi_{2}, \cdots, \quad \phi_{m}\right\}, \min T(\phi)=\max _{i=1,2, \cdots, n} T\left(\phi_{j}\right)
$$

\subsubsection{Solution of the Model}

The solution for the model 3.7 is completely similar to the method of calculating the single objective model in 3.1. We might as well suppose that $t_{i 1} \leq t_{i 2} \leq \cdots \leq t_{i q} \leq T$, then solve the critical subscript $p_{j}$ of $X_{i_{1} j}, X_{i_{2} j}, \cdots, X_{i_{q} j}$ about $X_{j}$, and next make $t_{1} \leq t_{2} \leq \cdots t_{n} \leq T$. Whereas, if there exist $p_{j}$, the critical subscript of $X_{1 j}, X_{2 j}, \cdots, X_{n j}$ about $X_{j}$, in this case, $\phi_{j}=\left\{\left(P_{1}, X_{1 j}^{\prime}\right),\left(P_{2}, X_{2 j}^{\prime}\right), \cdots,\left(P_{p_{j}-1}, X_{p_{j}-1}^{\prime}\right),\left(P_{p_{j}}, X_{j}-\sum_{i=1}^{p_{j}-1} X_{i j}\right)\right\} \quad$ and $\quad$ satisfying $T\left(\phi_{j}\right)=t_{p_{j}}$.

So, when $j=1,2, \cdots, m$, there respectively exist $p_{1}, p_{2}, \cdots, p_{m}$ about $X_{j}$. Then we can get the multiple supplies emergency distribution scheme as follows:

$$
\phi=\left[\begin{array}{cccc}
X_{11} & X_{12} & \cdots & X_{1 m} \\
X_{21} & X_{22} & \cdots & X_{2 m} \\
\vdots & \vdots & \vdots & \vdots \\
X_{p_{1}-1,1} & X_{p_{2}-1,2} & \cdots & X_{p_{m}-1, m} \\
X_{1}-\sum_{i=1}^{p_{1}-1} X_{i 1} & X_{1}-\sum_{i=1}^{p_{2}-1} X_{i 2} & \cdots & X_{1}-\sum_{i=1}^{p_{m}-1} X_{i m} \\
0 & 0 & \cdots & 0 \\
\vdots & \vdots & \vdots & \vdots \\
0 & 0 & 0 & 0
\end{array}\right]
$$

At the same time, there is $T(\phi)=\max _{j=1,2, \cdots, m} t_{p}$.

Because of the complexity of the model data, solution process needs to calculate with the help of computer program, to find out the optimal method. In this section, the paper firstly discusses the mathematical model and calculation method of the disaster relief logistics system, then builds a optimization model with the goal of " distribution time shortest" under the constraints of transport capacity. finally, solve by the computer. 


\section{Case Analysis}

\subsection{Transport without Capacity Constrains}

First, calculate the optimal distribution scheme on analysis of Transport without capacity constrains. In this case, we suppose the disaster occurs in Tianjin, 10000 tents are badly needed, and they are required to be delivered within 15 hours. In the measuring process, we stipulate the method of transport is highway transportation; the average speed is $50 \mathrm{~km} / \mathrm{h}$. Under the assumptions of the above, calculate the distance and distribution time from each region to disaster site, the result is shown in table 2.

Table 2 Distance and distribution time from suppliers to disaster site

\begin{tabular}{cccc}
\hline \multirow{2}{*}{ Number } & $\begin{array}{c}\text { Storage site-- } \\
\text { Disaster site }\end{array}$ & Distance (kilometer) & Time (hour) \\
\hline 1 & Tangshan--Tianjin & 150 & 3 \\
2 & Shijiazhuang--Tianjin & 550 & 11 \\
3 & Zhejiang-Tianjin & 1150 & 23 \\
4 & Henan-Tianjin & 1350 & 27 \\
5 & Nanchang--Tianjin & 1600 & 32 \\
6 & Hunan-Tianjin & 2050 & 41 \\
7 & Wuhan--Tianjin & 1250 & 25 \\
8 & Xining--Tianjin & 1500 & 30 \\
9 & Xi'an--Tianjin & 1400 & 28 \\
10 & Yinchuan--Tianjin & 1250 & 25 \\
\hline
\end{tabular}

Constraints are single mode of transportation and no capacity constraints. Take the data in table 2 into formula 3.3, and run the computer program in Turbu $C$, the optimal results are as follows:

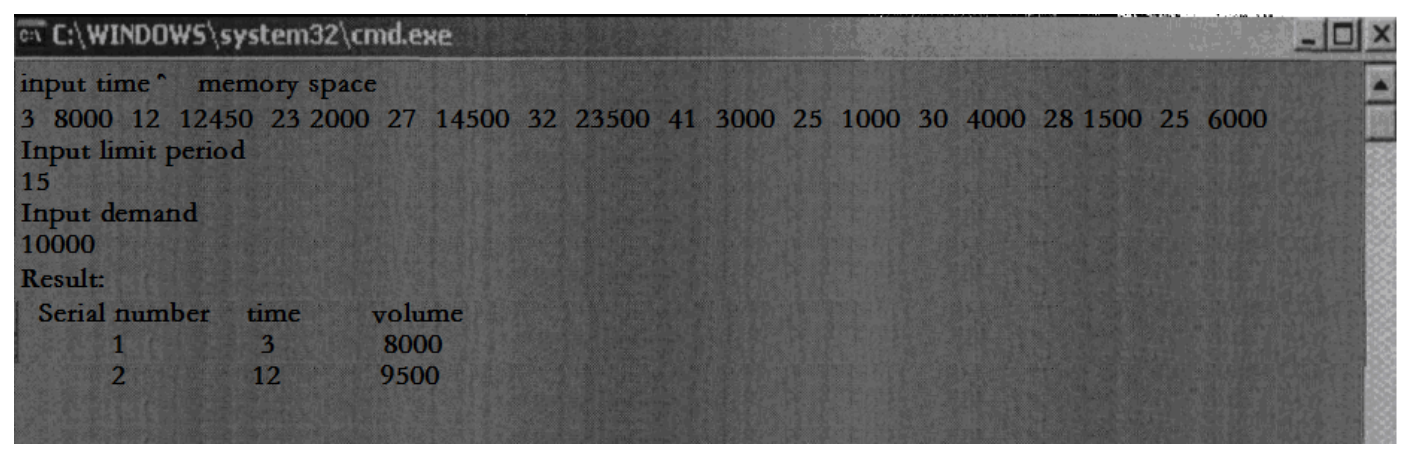

Figure 3. The Optimal Results

It can be known from the above picture that the optimum distribution scheme is: 8000 in Tangshan and 9500 in Shijiazhuang. Through the analysis of the best solutions under the condition of no capacity constraints, getting the total cost of time is 11 hours. This question researched at here is relatively simple, and it can also be reduced to the minimum distance problem, because the traffic volume is enough, and other conditions are assumed constant. 


\subsection{Transport with Capacity Constrains}

Considering the transportation capacity constraints, each place has a certain supplies reserves, the time limit is still 15 hours, and the demand quantity for tent is 10000. The comparison about the storage and transport capacity of each place is shown in table 3 .

Table 3. Storage and Transport Capacity Constrain of Tent

\begin{tabular}{|c|c|c|c|c|c|c|c|c|c|c|}
\hline Number & 1 & 2 & 3 & 4 & 5 & 6 & 7 & 8 & 9 & 10 \\
\hline $\begin{array}{c}\text { Storage } \\
\text { site }\end{array}$ & TangShan & $\begin{array}{c}\text { ShiJia } \\
\text { Zhuang }\end{array}$ & $\begin{array}{l}\text { Zhe } \\
\text { jiang }\end{array}$ & $\begin{array}{l}\mathrm{He} \\
\text { nan }\end{array}$ & $\begin{array}{c}\text { Nan } \\
\text { Chang }\end{array}$ & $\begin{array}{c}\mathrm{Hu} \\
\mathrm{Nan}\end{array}$ & WuHan & $\begin{array}{c}\mathrm{Xi} \\
\mathrm{Ning}\end{array}$ & $\begin{array}{l}\mathrm{Xi} \\
\mathrm{An}\end{array}$ & $\begin{array}{c}\text { Yin } \\
\text { Chuan }\end{array}$ \\
\hline $\begin{array}{l}\text { Transport } \\
\text { capacity }\end{array}$ & 6500 & 6000 & 3000 & 2000 & 9000 & 5500 & 4000 & 2500 & 2500 & 5000 \\
\hline $\begin{array}{l}\text { Storage } \\
\text { quantity }\end{array}$ & 7050 & 14400 & 6000 & 2000 & 25000 & 8500 & 1000 & 500 & 1500 & 3000 \\
\hline
\end{tabular}

Take the data in table 3 into formula 3.7, and run the computer program in Turbu $C$, the running process and optimal results are as follows:

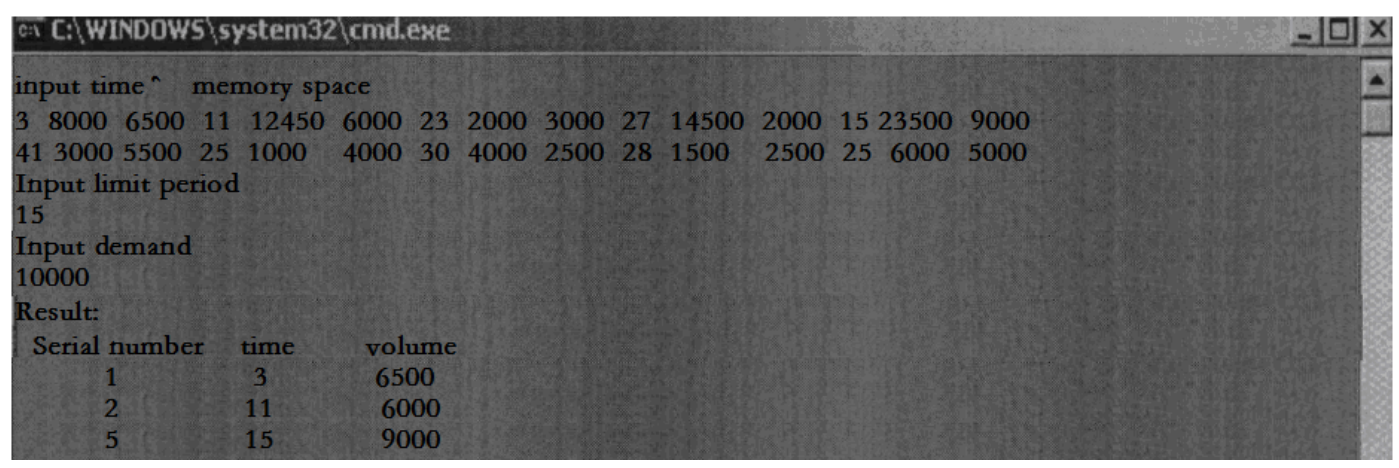

Figure 4. The Running Process and Optimal Results

It can be known from the above picture that the optimum distribution scheme is: 6500 in Tangshan, 6000 in Shijiazhuang and 9000 in Nanchang, the total cost of time is 17 hours.

By comparing the two experimental results of transport without capacity constrain and with capacity constrain, it seems that under the same time constraints, transport with capacity constraints is relatively complicated, because considering the constrains of capacity and storage, the organizers need to consider the situation of repeatedly distribution. In actual measuring process, there are not only these factors can influence the results, in this case we just neglect the other factors. Besides, the transport capacity is the key factor, which must be taken into account in the calculation process. Because of the complexity of the calculation process, the computer program is greatly needed to quickly accurate the optimal the computer program scheme.

\section{Conclusion}

This paper studies the supplies distribution model of multiple suppliers and single demand site in emergency. The single supplies and multiple supplies without and with the transport capacity constrains are discussed respectively. In the process of 
building model, this paper only consider single method of transportation and single target, that is to say, this paper take the time shortest as the unique goal.

On the basis of studying single target and static system, emergency supplies distribution model of multiple target and dynamic conditions is discussed in further. Multiple objective programming mainly takes economy as the second goal, and then builds the distribution model. Guaranteeing the supplies safety of disaster relief logistic system under dynamic conditions mainly by building supplies distribution model of object-oriented, when one or a few conditions change in the system, an alternative scheme is quickly started to implement the quick response of disaster relief logistic system.

\section{References}

[1] Linet Ozdamar, Ediz Ekinci. Emergency Logistic Planning in Nature Disasters. Annals of Operations Research.. 2004. (129): 217-245.

[2] Philip T.Evers. FIlling Customer Orders from Multiple Locations: A Comparison of Pooling Methods[J]. Journal of Business Logistics, 1999, 20(1).

[3] S.M.Hon-Minh, S.M.Disney\&MM.Naim. The Dynamics of Emergency Transshipment Supply Chains [J]. International Journal of Physical Distribution\&Logistics Management, 2000, 30(9).

[4] Takeo Yamad. A network flow approach to a city emergency evacuation planning [J]. International Journal of Systems Science, 1996, 27(10): 931-936.

[5] Culay Barbaroso glu a, Linet Ozdamarb and Ahmet Cevik b, An interactive approach for Hierarchical analysis of helicopter logistics in disaster relief operations[Jl, European Journal of Operational Research. 2000. 140: 118-133.

[6] Sun M H, Aronson J E. A tabu search heuristics procedure for the fixed charge Transportation problem[J]. European Journal of operation Research, 1998, 106(3): 441-456.

[7] Ali Hand Sei-Chang Oh, Formulation and solution of a multi-commodity multi-modal network flow model for disaster relief operation. Transportation Research-A-. 1996. 30(3): 231-250.

[8] Gwo-Hshiung Tzeng, Hsi-Jung Cheng and Tsung Dow Huang. Multi-objective optimal planning for Designing relief delivery systems. Transportation Research PartE[M], 2007, 43(6): 673-686.

[9] Yi Wei, Aran Kumar. Ant colony optimization for disaster relief operations.Transportation Research PartE[M], 2007, 43(6): 660-672.

[10] Kaan Ozbay, Weihua Xiao, Cem Iyigun, Melike Baykal-Gursoy. Probabilistic programming models for response vehicle dispatching and resource allocation in traffic incident management[R]. Rutgers University, I\&SE-Working paper, 2004. 4-14.

\section{Authors}

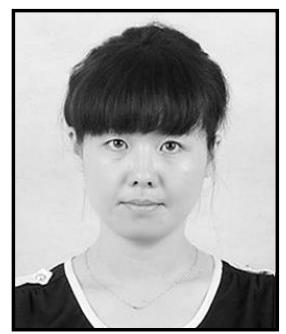

CHEN Ling-ling, female, is born in 1984 in Tangshan, Hebei province. She is wuhan university of science and doctoral students. Now she works at Yanching Institute of Technology as a lecturer. Her research direction is transportation logistics management. 